\title{
Low-Voltage Continuous Electrospinning Patterning
}

\author{
Xia Li, ${ }^{\dagger}$ Zhaoying Li, ${ }^{\ddagger}$ Liyun Wang, ${ }^{\S}$ Guokun Ma, ${ }^{\prime}$ Fanlong Meng, ${ }^{\dagger}$ Robyn H. Pritchard, ${ }^{\dagger}$ \\ Elisabeth L. Gill, ${ }^{+}$Ye Liu, ${ }^{\ddagger}$ and Yan Yan Shery Huang*, \\ ${ }^{\dagger}$ Cavendish Laboratory, University of Cambridge, JJ Thomson Avenue, Cambridge CB3 0HE, United Kingdom \\ ${ }^{\ddagger}$ Department of Engineering, University of Cambridge, Trumpington Street, Cambridge, CB2 1PZ, United Kingdom \\ ${ }^{\S}$ Department of Food Science and Technology, Jiangnan University, Wuxi 214122, China \\ "State Key Laboratory of Electronic Thin Films and Integrated Devices, University of Electronic Science and Technology of China, \\ Chengdu 610054, China
}

\section{Supporting Information}

\begin{abstract}
Electrospinning is a versatile technique for the construction of microfibrous and nanofibrous structures with considerable potential in applications ranging from textile manufacturing to tissue engineering scaffolds. In the simplest form, electrospinning uses a high voltage of tens of thousands volts to draw out ultrafine polymer fibers over a large distance. However, the high voltage limits the flexible combination of material selection, deposition substrate, and control of patterns. Prior studies show that by performing electrospinning with a well-defined "near-field" condition, the operation voltage can be decreased to the kilovolt range, and further enable more precise patterning of fibril structures on a planar surface. In this work, by using solution dependent "initiators", we demonstrate a further lowering of voltage with an ultralow voltage continuous electrospinning patterning
\end{abstract}
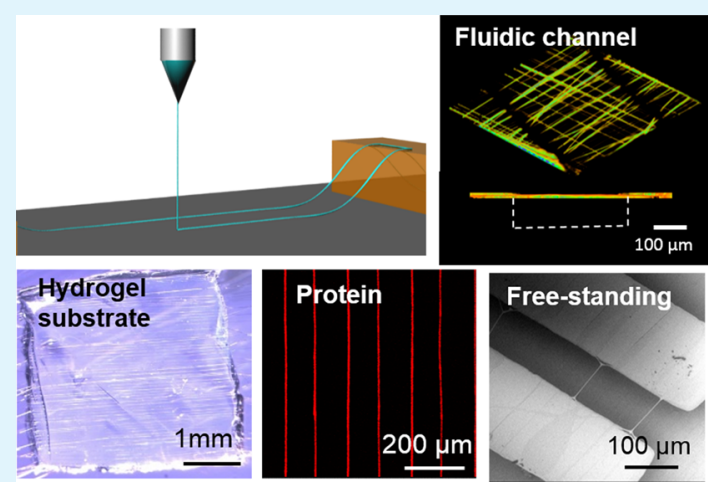

(LEP) technique, which reduces the applied voltage threshold to as low as $50 \mathrm{~V}$, simultaneously permitting direct fiber patterning. The versatility of LEP is shown using a wide range of combination of polymer and solvent systems for thermoplastics and biopolymers. Novel functionalities are also incorporated when a low voltage mode is used in place of a high voltage mode, such as direct printing of living bacteria; the construction of suspended single fibers and membrane networks. The LEP technique reported here should open up new avenues in the patterning of bioelements and free-form nano- to microscale fibrous structures.

KEYWORDS: electrospinning, direct writing, bacteria patterning, 3D architectures, biomembrane, biofabrication, nanofibre

\section{INTRODUCTION}

Applications of micro-nanofibrous structures have become vital to a number of emerging technologies such as fiber-based sensors, ${ }^{1-3}$ filtration membranes, ${ }^{4-6}$ batteries and energy storage $^{7-9}$ and a range of bioengineering technologies such as tissue scaffold implants, ${ }^{10-12}$ drug delivery ${ }^{13}$ and wound dressing. ${ }^{14}$ Electrospinning is regarded as one of the most widespread techniques offering rapid, inexpensive, and relatively large-scale fabrication for fibrous structures. ${ }^{15-18}$ Conventional electrospinning processes operate at high voltages (more than tens of kilovolts) and creates randomly coiled fibers because of the inherent bending instability of the fiber jet. ${ }^{19}$ To fabricate refined, well-controlled geometrical structures, researchers have developed several experimental techniques (such as electrospinning with a modified electric field $^{20}$ and using a rotating cylinder as a collector ${ }^{21}$ ) to allow aligned fibrous construction with limited flexibility and configurations. Precise control of fiber placement is essential in determining the functionalities of biomimetics and electronic devices. ${ }^{17}$ However, this is difficult to achieve using highvoltage techniques. Recent development in melt electro- spinning successfully demonstrates precisely controlled microto nanoscale fibril structures in $3 \mathrm{D} .^{22,23}$ This technique sees great potential to complement the present additive manufacturing techniques, though it is limited to thermoplastic materials. Significant improvement on controllable patterning has been made by mechano-electrospinning ${ }^{24-26}$ where not only fibers but also beaded deposition can be made. ${ }^{26}$ In particular, nearfield electrospinning (NFES) techniques ${ }^{27,28}$ enable the patterning of a variety of polymer-solvent combinations with some reduced flexibility in patterning precision due to a lower viscous jet. Operating at a closer distance (e.g., $\sim 1 \mathrm{~mm}$ ) over a moveable stage, the applied voltage of NFES is usually in the kilovolt range. Various functional materials have been patterned by NFES to date, such as for electrical, ${ }^{28,29}$ optical, $^{30}$ and biological $^{31}$ applications. Two-dimensional patterns with enhanced complexity have been deposited on silicon wafers, by varying the experimental setup or processing parameters. ${ }^{32}$

Received: June 27, 2016

Accepted: November 3, 2016

Published: November 3, 2016 

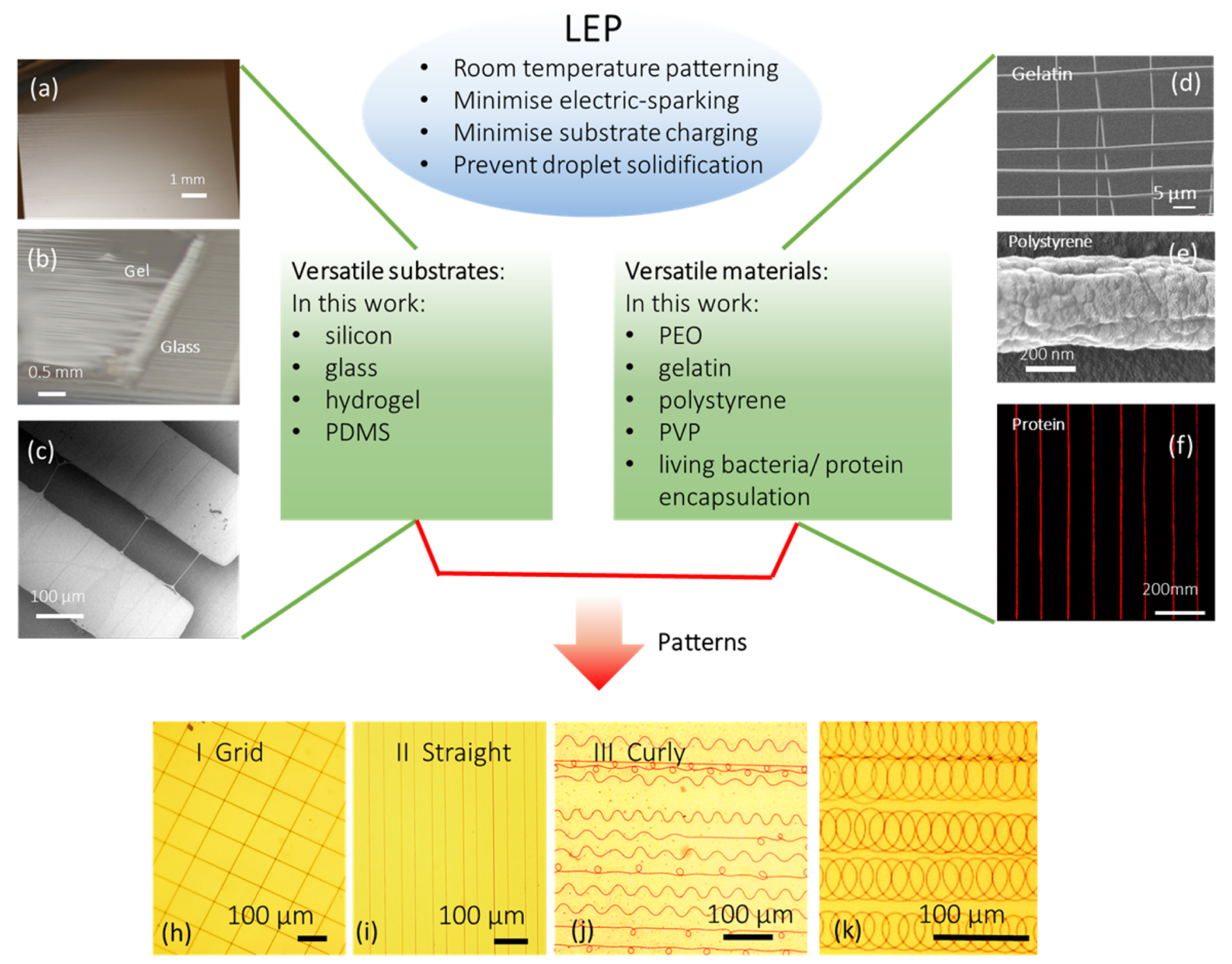

Figure 1. Overview of the attributes of LEP as a versatile material, versatile substrate deposition technique. (a-c) Versatile deposition substrates, such as PEO fibers on glass; gelatin fibers first deposited on glass and then on top of an agarose gel; and suspending gelatin fiber bridging two PDMS pillars. ( $d-f)$ versatile depositing materials, such as gelatin in the form of a suspending cross-lattice; polystyrene fibers with surface roughness formed from a rapidly solidifying solution, where the LEP helps to solve the drying issue during printing; and fluorescent proteins encapsulated into PEO fibers. ( $\mathrm{h}-\mathrm{k}$ ) Various forms of patterns can be achieved similar to the conventional NFES techniques, such as grids, straight lines, and curly lines resulting from the mismatch between jet speed and stage speed.

Current improvements still need to be seen are associated with the precise deposition control in a substrate-free form, or in a $3 \mathrm{D}$ configuration. However, this is limited by a number of existing technical constraints. First, while NFES enables a much reduced voltage $(1-2 \mathrm{kV})$ compared to conventional electrospinning (tens of kilovolts ${ }^{33}$ ), such a voltage level may still induce degradation and damages to the inherent electronic, biological or pharmaceutical functionalities associated with individual fibers. Second, as most NFES are operating at an average field strength in the order of $\mathrm{MV} / \mathrm{m}$ (close to the dielectric strength of air), electric discharge may occur which can damage any delicate depositing substrates. Our experience in using NFES also shows that direct patterning on insulating substrates such as glass or PDMS are limited at a thickness of $\sim 1 \mathrm{~mm}$. Finally, because precise deposition requires a low solution feed rate, this often results in the drying of the droplet exposed, blocking the output of the flow feeding, and thus the deposition process cannot be continuously sustained. In an industrial level, further undesirable effects arise from the high electric field are such as deviation of the multijet electro- spinning and fiber collection difficulties caused by the high electric field surrounding the needles. ${ }^{34}$

Herein, we report an ultralow voltage continuous electrospinning patterning (LEP) technique, with typical operation voltages at $100 \mathrm{~V} \mathrm{DC}$, in a template free route. Under this electrospinning mode, the combination of the lateral mechanical stretching force and the electrostatic field focusing effects facilitates the initiation of the polymeric jet, mitigating the various fiber deposition challenges inherent with a high voltage technique as described above. Precisely controlled single fiber geometries are patterned over large areas at a line speed up to $150 \mathrm{~mm} / \mathrm{s}$. With the low application voltage, designed patterns can be fabricated on a range of substrates, from conducting to insulating in nature, and from hydrogels to solids. Furthermore, a versatile range of solutions can be deposited with voltages as low as $50 \mathrm{~V}$. We demonstrate solution systems based on a high boiling point organic solvent, a low boiling point organic solvent, and an aqueous solvent, with common molecular weight polymers of polystyrene (PS), polyvinylpyrrolidone (PVP), gelatin, and poly(ethylene oxide) 

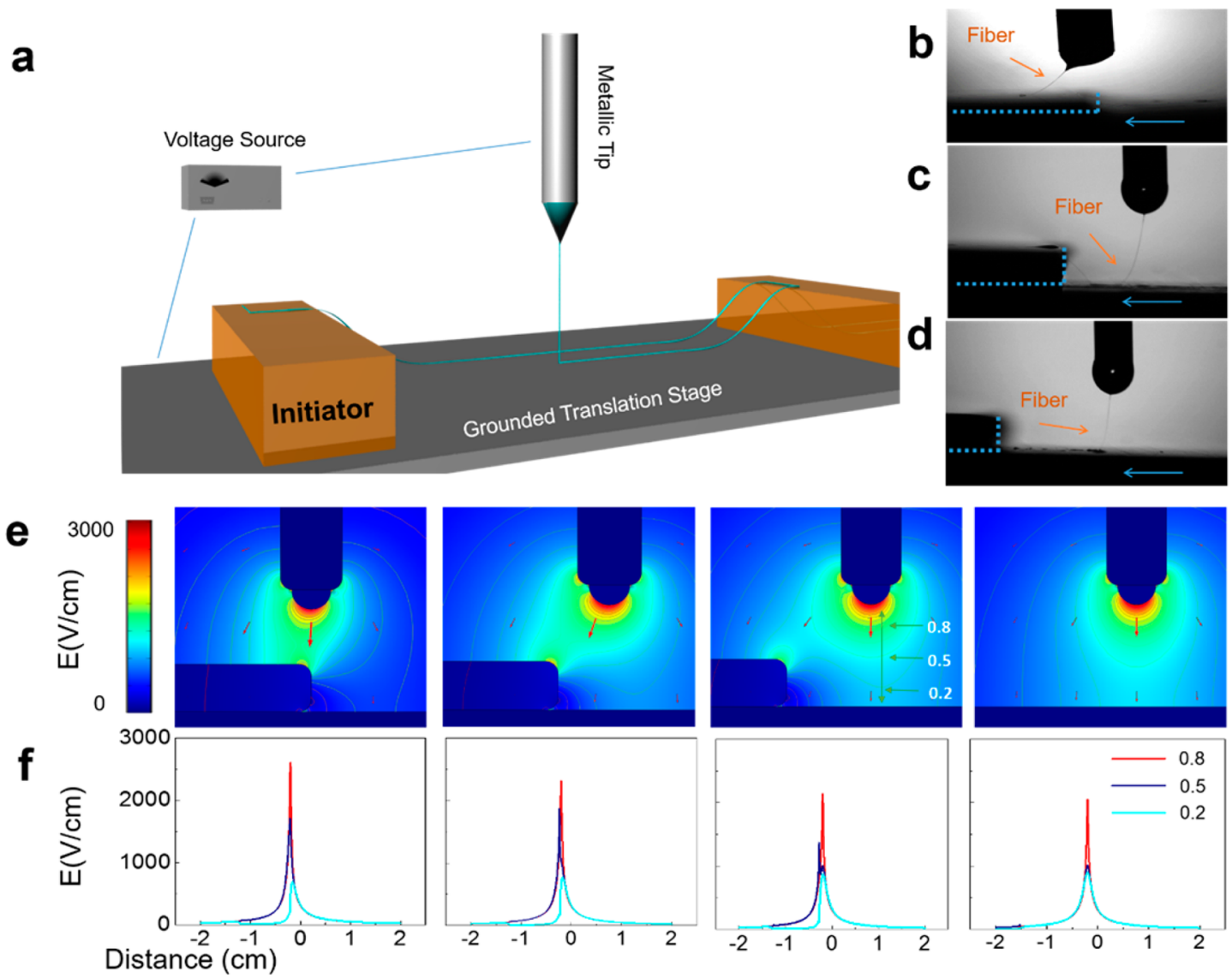

Figure 2. (a) Schematic figure illustrates the configuration of the LEP setup. (b-d) Frames taken by a high-speed camera during the LEP process. (e) Simulation on the electric field distribution with the translation of the initiator relative to the jetting tip. Numeric curves in (f) reflect the corresponding electric field change following the stage movement in e. The three lines in each plot are $z$ distances (in mm) sampled from the grounded collector $(z=0)$ toward the apex of the droplet. One can clearly identity an electric field enhancement effect caused by the initiator, through comparing the peak electric field values (e.g., the red curves) across the three tip-initiator positions.

Table 1. Solution Conductivity versus Voltage Threshold at a Fixed Working Distance $(1 \mathrm{~mm})$ during Electrospinning without and with Initiator ${ }^{a}$

\begin{tabular}{|c|c|c|c|c|c|c|}
\hline polymer & $\begin{array}{l}\operatorname{mol~wt} M_{\mathrm{w}} \\
\quad(\mathrm{Da})\end{array}$ & $\begin{array}{l}\text { concentration (wt/ } \\
\mathrm{wt})\end{array}$ & $\begin{array}{l}\text { conductivity }(\mu \mathrm{S} / \\
\mathrm{cm})\end{array}$ & $\begin{array}{l}\text { voltage threshold (V) (without } \\
\text { initiator) }\end{array}$ & $\begin{array}{c}\text { voltage threshold (V) (with } \\
\text { initiator) }\end{array}$ & $\begin{array}{l}\text { nanofiber } \\
\text { morphology }\end{array}$ \\
\hline \multirow[t]{4}{*}{$\mathrm{PEO}$} & \multirow[t]{4}{*}{400000} & 0.03 & 106.4 & $\mathrm{X}$ & $1000(\mathrm{~g} / \mathrm{s})$ & $\begin{array}{l}\text { beads, } \\
\text { discontinuous }\end{array}$ \\
\hline & & 0.04 & 100 & 2000 & $1000(\mathrm{~g} / \mathrm{s})$ & $\begin{array}{l}\text { beads, } \\
\text { discontinuous }\end{array}$ \\
\hline & & 0.05 & 99.7 & $1000-2000$ & $50(\mathrm{~g} / \mathrm{s})$ & beads, continuous \\
\hline & & 0.06 & 99.7 & 600 & $50(\mathrm{~g} / \mathrm{s})$ & $\begin{array}{l}\text { uniform, } \\
\text { continuous }\end{array}$ \\
\hline \multirow[t]{4}{*}{ PVP } & \multirow[t]{4}{*}{600000} & 0.15 & 6.3 & $\mathrm{X}$ & $\mathrm{X}$ & $\mathrm{X}$ \\
\hline & & 0.2 & 6.4 & 2000 & 1000 s only & $\begin{array}{l}\text { beads, } \\
\text { discontinuous }\end{array}$ \\
\hline & & 0.25 & 6.5 & 1000 & $70 \mathrm{~s}$ only & $\begin{array}{l}\text { uniform, } \\
\text { continuous }\end{array}$ \\
\hline & & 0.30 & 4.5 & 1000 & $70 \mathrm{~s}$ only & $\begin{array}{l}\text { uniform, } \\
\text { continuous }\end{array}$ \\
\hline \multirow[t]{3}{*}{ PS } & \multirow[t]{3}{*}{280000} & 0.2 & 0.35 & $\mathrm{X}$ & $\mathrm{X}$ & $\mathrm{X}$ \\
\hline & & 0.25 & 0.30 & 1200 & $50 \mathrm{~s}$ only & $\begin{array}{l}\text { uniform, } \\
\text { continuous }\end{array}$ \\
\hline & & 0.3 & 0.19 & 1200 & $50 \mathrm{~s}$ only & $\begin{array}{l}\text { uniform, } \\
\text { continuous }\end{array}$ \\
\hline
\end{tabular}

${ }^{a}$ The glass slides (denoted with a symbol ' $\mathrm{g}$ ') and silicon wafer (denoted with a symbol 's') were used as insolating and conductive initiators, respectively, in order to compensate or match the solution conductivities. Other insulating (e.g., plastics) or conducting materials (e.g., metallic plates) can also be used. To obtain consistent comparison from the initiators for fiber onset, we kept the thickness of the initiators for both glass and silicon wafers at $\sim 0.5 \mathrm{~mm}$. The symbol ( $/ \mathrm{g}$ ) represents that both silicon wafers or glass slides can be used as initiators; (s only) means only silicon wafer is used as the initiator, mainly because of the low conductivity of the solution. 
a

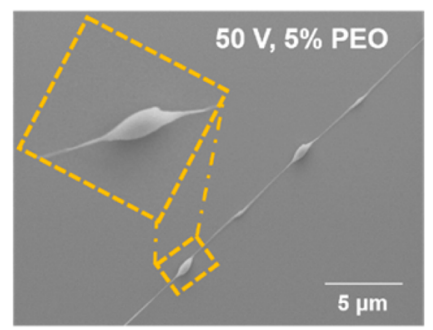

b

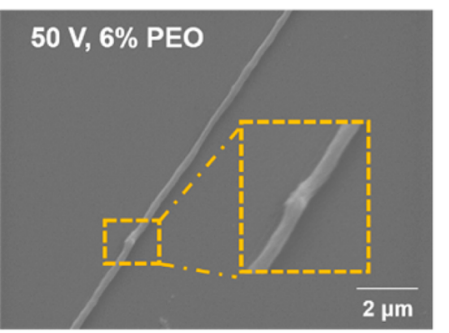

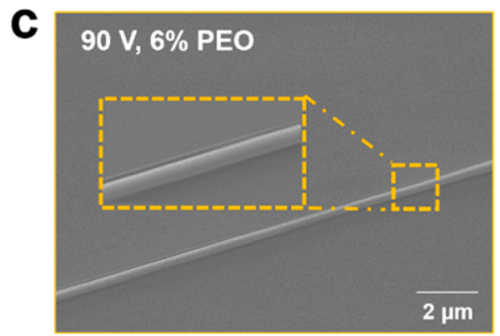
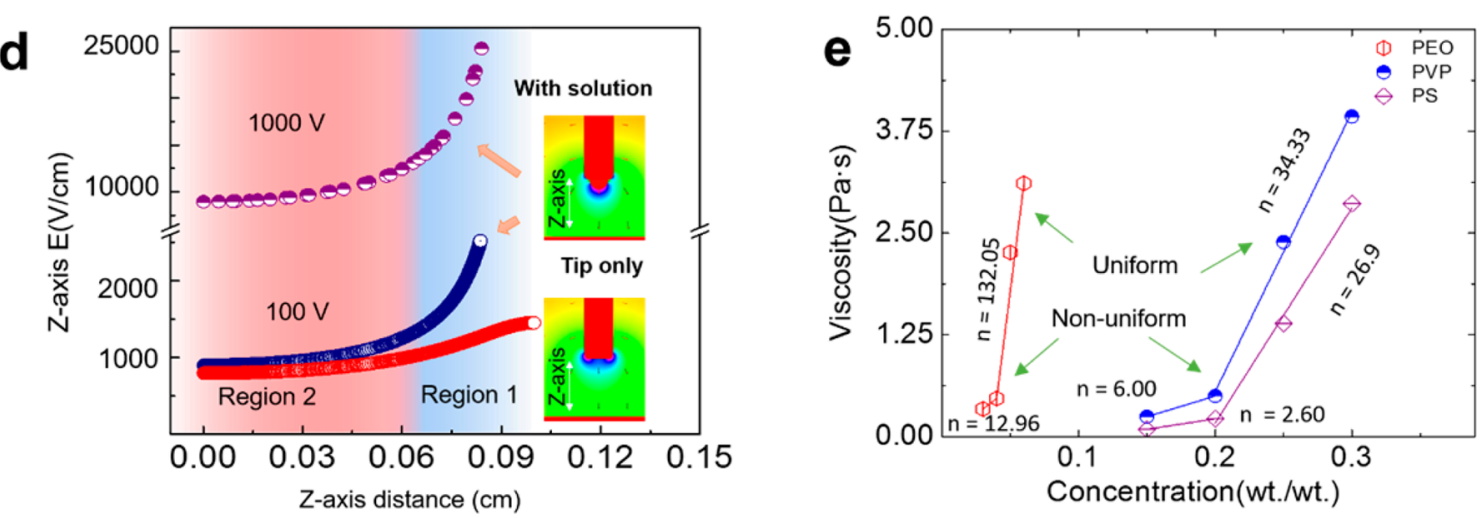

Figure 3. SEM images of PEO fibers fabricated through LEP. (a) 5\% PEO-water solution through $50 \mathrm{~V}$ giving a beads-on-string feature, where the fiber is not attached to the substrate. (b) 6\% PEO-water solution with $50 \mathrm{~V}$ giving a straight fiber with twisted morphology. (c) 6\% PEO-water solution with $90 \mathrm{~V}$, showing a uniform fiber evenly attached to the substrate. (d) Direct comparison by the simulation of electric field distribution for a voltage application of 100 and $1000 \mathrm{~V}$, using a model with/without the consideration of the droplet effect. (e) Viscosity profiles of different polymeric solutions used for this study.

(PEO). LEP also allows 3D construction and patterning living bacteria with reduced electrostatic influence. Our experiments show the universality of LEP technique, demonstrating its potentials in a number of emerging fields.

\section{RESULTS AND DISCUSSION}

The proposed advantages and applications of LEP system are summarized in Figure 1. The key to reducing the operational voltage is to minimize the threshold voltage required for jet initiation. In established NFES processes, a voltage of $1-2 \mathrm{kV}$ is applied to a metallic tip ${ }^{35}$ resulting in an electrostatic force on the suspended droplet. The internal charge repulsion leads to the deformation of the droplet into a Taylor cone, ${ }^{36}$ which in turn initiates fiber jetting. ${ }^{28}$ If continuous electrospinning is to occur under a reduced voltage, it is essential to sustain a comparable electric field around the local position of the droplet to overcome the surface tension. Unlike conventional far-field electrospinning, at the close distances employed in NFES, the suspended droplet volume becomes non-negligible. ${ }^{17,37}$ Thus, it is undesirable to decrease the electrospinning distance to reduce the voltage because the suspending droplet may cause electric sparkling when the droplet is being stretched to bridge the tip and the deposition substrate. ${ }^{37}$ Furthermore, because the electric field rapidly decreases from the tip toward the grounded collector, it is therefore difficult to initiate fiber jetting with voltages below $500 \mathrm{~V}$, which is especially the case for solutions of low conductivities.

Electrospinning in Low Voltages. To overcome the difficulties of continuous electrospinning under low voltages, LEP incorporates a lateral mechanical stretching force, in conjunction with an electrostatic field focusing effect, to initiate polymeric jetting. The combined effects can be achieved by utilizing "initiators" on either sides of the patterned route, shown in Figure 2a. The selection of initiators (e.g., conducting or insulating) depends on the solution conductivity, see Table 1 and the Experimental Section. It is of note that after initial solution charging, a voltage reduction to zero can still yield temporary fibers production. However, such fiber production cannot be self-sustained. In order to obtain continuous deposition, voltages have to be continuously applied during the fiber patterning process.

Sequential images recorded by a high-speed camera in Figure $2 \mathrm{~b}-\mathrm{d}$ show the electrospinning patterning process: first, the droplet is significantly deformed above the initiator, with a thick fiber ejecting from the apex of the droplet; in the second frame, as the initiator moves away from the tip, the droplet forms a cone shape and the fiber exhibits thinning, directed toward the depositing substrate. We suggest this process assists the redistribution of the surface charges on the droplet, providing a local region that contains higher charges at the droplet surface. ${ }^{38}$ The initiators also change the global electric field distribution; where comparatively more concentrated local electric field is formed when the initiators are closer to the tip, as confirmed by simulation shown in Figure 2e. Figure $2 \mathrm{f}$ quantifies the corresponding electric field intensity along the $z$ axis from the grounded collector $(z=0 \mathrm{~mm}$,) to a level just below the droplet apex (i.e., $z=0.8 \mathrm{~mm}$ ) above the collector, as a function of substrate position. The spatial dependence of the electric field is modified by the movement of the initiators and the substrate, with a concentrated electric field between the tip and the initiator edge. With an applied voltage of $100 \mathrm{~V}$, local focusing of the electric field can reach as high as $2500 \mathrm{~V} / \mathrm{cm}$ when the initiator is close to the tip position. As the substrate moves to the center, the highest value field strength decreases to $2000 \mathrm{~V} / \mathrm{cm}$ and the global electric field distribution becomes symmetrical. Under this condition, fibers can then be deposited 
accurately with prescribed patterns. By adjusting the collector speed and the flow rate of the polymer solution, a steady-state is achieved which enables continuous electrospinning. During this process, the suspending droplet is refreshed by the initiators; simultaneously, the volume of the droplet remains constant by the adjusted polymer flow rate. The above procedure with cyclic initiation thus enables a continuous electrospinning and direct-patterning process.

On the basis of the above experimental and simulation results, we suggest that the presence of the initiator can assist the onset of fiber jetting, as well as enhancing the fiber thinning process. First, the configuration of the initiator helped to strengthen the localized electric field at regular times (e.g., approximately every few seconds depending on the sample size), when the sample moved to the position near the initiator as shown in Figure 2f. Second, the initiator provides a mechanical lateral stretching force when the initiator is moved away from the droplet, see Figure $2 \mathrm{~b}$. This mechanical thinning effect resembles that of the fiber drawing mechanism used in other fibrous patterning techniques(STEP) $)^{39-41}$ where fibers were drawn without a voltage application. In $\operatorname{STEP}^{39}$ and the subsequent work, only organic solvent-based solutions were demonstrated. Here, since an electrospinning mechanism (Taylor cone) was employed for fiber initiation, a variety of solvent-material combinations can be used, which will be demonstrated in the following section. Third, for highly volatile solvents, one can actually use a higher flow rate to keep the droplet refreshed without affecting the patterning process. This is because, the droplet would be undergone the most deformation at the initiator positions. Hence, any excessive increase in droplet volume would be drawn to and deposited at the position of the initiators, rather than affecting the fiber patterning on the targeted substrate.

The competition of Coulomb's force, solution viscosity and solution surface tension determines both the fiber trajectory and the single fiber morphologies during an electrified jet printing process. ${ }^{15,19}$ In NFES, the ejected polymer stream avoids several bending instabilities, as well as environmental disturbance including the lateral aerodynamic resistance as the fiber falling toward the collector. This is because the localized electric field is sufficient to dominate over the aerodynamic disturbance. In comparison, LEP shows distinct fiber morphologies depending on the voltage application and the solution concentration (see Figure $3 a-c$ ). We suggest this to be attributed to the fact that the aerodynamic resistance is of similar magnitude to the electrostatic force. To evaluate the relative difference between a NFES and a LEP processes, Figure $3 \mathrm{~d}$ compares the electric field focusing effect introduced at the metallic tip with a $1 \mathrm{kV}$ operation voltage (i.e., a standard NFES process), and a $100 \mathrm{~V}$ operation voltage (an LEP process) at a typical working distance of $1 \mathrm{~mm}$. It is shown that the electric field intensity decreases dramatically at the surface of the collector. This simulation results suggest that the fiber trajectory can be influenced by aerodynamic disturbance. Next, we will discuss the effect of solution viscosity on fiber morphology (see Figure $3 a-c$, e). With a low solution viscosity (a low polymer concentration), continuous fibers fabricated using a 5\% PEO-water solution (2.3 Pa s) under $50 \mathrm{~V}$ exhibits a beads-on-string structure, which slightly differs from the existing work showing uniform fibers ${ }^{42}$ with similar viscosity, probably because of the difference in the PEO molecular weight used. Mechanisms for forming morphologically similar beaded structures have been proposed in far field electrospinning. ${ }^{43}$ It was postulated that fibers formed from lower polymer concentrations were more prone to beaded structures because of the prolonged solidification time, and thus the fiber morphology was a combined effect of surface tension and the viscoelastic property of the solution. ${ }^{44,45}$ In comparison, fibers formed from solutions of high concentrations could solidify faster, and tend to be uniform in thickness and were less influenced by the Rayleigh instability. ${ }^{44,45}$ In our LEP case, although the duration needed for fibers to reach a collector is reduced because of a small working distance, the fibers could still exist in a semiliquid state when deposited (also evidenced from previous studies $\left.{ }^{28,31}\right)$. This is in contrary to the far-field electrospinning case, where the fibers were allowed sufficient time to solidify during traveling to the collector. Thus, we postulate that for LEP, the formation of beads-on-string structure can be explained with a similar mechanism proposed for far-field electrospinning because of Rayleigh instability; ${ }^{43}$ but instead this modulation would take place on the substrate rather than in air. ${ }^{44,45}$ Because the liquid fiber would interact with the substrate surface, both the fiber solidification time as well as the wetting between the substrate and the "wet" fiber can also affect the fiber morphology. Nonetheless, when the $\mathrm{PEO}-$ water solution concentration increases to $6 \%$, the fiber becomes smooth and uniform. Because of the weak electric field, the fibers exhibit twists and do not firmly attach to the substrate. As the applied voltage further increases to $90 \mathrm{~V}$, twist-free, straight, and firmly attached fibers are obtained. In this case, it is likely that the electrostatic force is large enough to overcome any lateral disturbance, giving a direct patterning process. Overall, the interplay between applied voltage, solution viscosity and aerodynamic effect governs the fiber morphology and the degree of fiber attachment onto a substrate. This will in turn determine the controllability of the deposited patterns.

Electrospinnability. The onset of fiber formation, though varied between different polymer and solvent combinations, greatly depends on the solution properties such as conductivity, surface tension, viscosity, as well as experimental parameters including applied voltage, tip shape, and working distance. ${ }^{15}$ In this work, we aim to establish general protocols that can be adapted to a wide range of polymers, using a selection of example polymers as summarized in Table 1 . Since nonnegligible droplet volume increased the electric field focusing effect significantly as opposed to a tip-only situation (shown in Figure 3d), we selected initiator materials based on the polymer solution conductivity used for electrospinning. Table 1 shows the conductivity value of PEO-water, PS-DMF, and PVPethanol solutions in different concentrations and their electrospinning performance. The conductivity of a polymer solution is strongly dependent on the nature of the polymer and its solvent but weakly dependent on the polymer concentrations within the electrospinnable regime. The conductivity of PEOwater, PVP-ethanol, and PS-DMF solution lies in the range between 95 and $107 \mu \mathrm{S} / \mathrm{cm}, 4.6$ and $5 \mu \mathrm{S} / \mathrm{cm}$, and 0.3 and 1 $\mu \mathrm{S} / \mathrm{cm}$, respectively. Therefore, because of the high conductivity of $\mathrm{PEO}-$ water solution, insulating initiators (glass slides) were used to assist jet initiation. For PS-DMF and PVP-ethanol solutions, conducting initiators (silicon wafers) were used because of the low solution conductivity.

The limiting polymer concentrations required for LEP were also investigated. Figure $3 \mathrm{e}$ shows the shear viscosities for the PEO $(\mathrm{Mw}=400 \mathrm{kDa})$ in water (aqueous), PS $(\mathrm{Mw}=280$ $\mathrm{kDa}$ ) in dimethylformamide (DMF) (a high boiling point organic solvent) and PVP $(\mathrm{Mw}=600 \mathrm{kDa})$ in ethanol (a low 

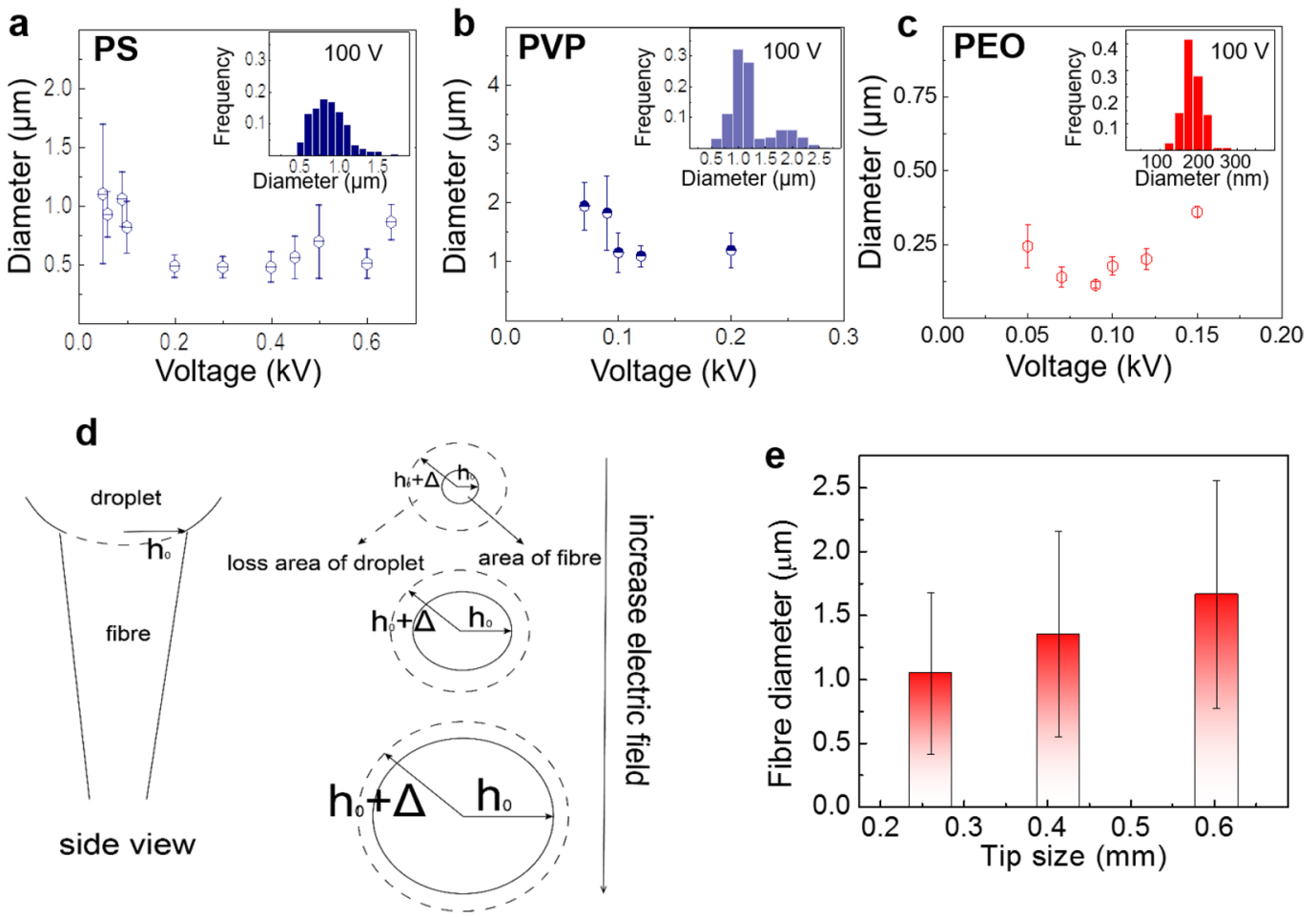

Figure 4. $(\mathrm{a}-\mathrm{c})$ Average fiber diameter with standard deviation dependence on the applied voltages for PS, PVP, and PEO. Inset: Normalized frequency of fiber diameter distribution at the specified voltage. (d) Scheme showing how the fiber diameter can be determined by the applied electric field by taking into account the effects of droplet deformation and fiber stretching. (e) Bar chart showing the influence of syringe tip size on the resulted fiber diameter, for a gelatin-aqueous solution.

boiling point organic solvent) solutions. Experimentally, we found that solutions with a low viscosity (i.e., 3-4\% PEOwater, 15-20\% PS-DMF, and 15-20\% PVP-ethanol) produce nonuniform fibers under low voltage electrospinning or conventional NFES. As the polymer solution concentration increases, more entanglements of the polymer chains are established, resulting in a sharp upturn of the solution viscosity. To demonstrate this trend, we applied linear regression to the viscosity vs concentration curves in the less-entangled and the more-entangled regimes separately, as shown in Figure 3e. The gradient of the 3-4\% PEO-water solution is 13.0, and increased to 132.0 for $4-6 \%$. Increasing the concentration of PS-DMF and PVP-ethanol solutions from 20 to $30 \%$, the gradient increased from 2.6 to 26.9 and 6.0 to 34.3 , respectively. The threshold of entanglement concentration for LEP associated with each polymer solution corresponds well with the electrospinnable polymer concentration. For example, PEO-water solution with $>5 \%$ polymer concentration $(\eta>$ $2.3 \mathrm{~Pa} \mathrm{~s})$, and PVP-ethanol solution with $>25 \%(\eta>2.4 \mathrm{~Pa} \mathrm{~s})$, could be electrospun into continuous fibers under the LEP with a typical voltage of $100 \mathrm{~V}$. In all, we suggest that for the polymers tested in our work, the operation of sub-100 V electrospinning requires the polymer solutions to reach certain concentrations (i.e., above 5\% for PEO-water, 25\% for PVPethanol, and $25 \%$ for PS-DMF $(\eta>1.4 \mathrm{~Pa} \mathrm{~s})$ ).

Next, we study the dependence of fiber width with respect to the applied voltage. For established NFES processes, it was found that the fiber width decreased as the applied voltage decreased. Chang et al. accounted this to the suspending droplet size which had pronounce effects on the fiber diameters due to the small gap distance between the tip and the collector. ${ }^{37}$ In this work, with a fixed electrospinning distance between the tip and the collector $(0.5 \mathrm{~mm})$, the dependence of the fiber width on the applied voltage is not monotonic, as shown in Figure $4 a-c$. In our experiment, we find that the radius of the fiber first decreases with the electric field, and then increases. Our findings differ from the scaling law relationship associated with the far-field electrospinning, which predicted that the fiber radius $h$, continuously decreases with increased electric field, $E$, in the form of $h \propto E^{-\alpha}$, with a power exponent $\alpha=1 / 4$ as found in previous works. ${ }^{46}$ The scaling law was mainly due to the electric stretching force imposed on the fiber jet. The above scaling law relationship is established based on the assumption that the size of droplet cone leading to fiber production, stays constant with an electric field. In reality, the cone size is also affected by the electric field, and this factor is pronounced for the near-field mode of electrospinning than the far-field mode. To account for the electric field influence on the cone size, and to explain our observation of the nonmonotonic dependence of fiber diameter on the electric field, we perform the following analysis. First for simplicity, we assume the shape of the droplet as a sphere, as shown in Figure 4d. With an increased electric field, the radius of the core will increase, due to electrostatic-repulsion in the droplet. The total energy change of the system for fiber formation, $\Delta F \approx-\pi \gamma\left(h_{0}+\right.$ $\Delta h(E))^{2}+2 \pi \sigma h_{0}$, includes two terms. The first term, $-\pi \gamma\left(h_{0}+\right.$ $\Delta h(E))^{2}$ is the surface energy change of the droplet cone, where $\gamma$ is the surface tension of the droplet, $h_{0}$ is the initial fiber radius; thus $\pi\left(h_{0}+\Delta h(E)\right)^{2}$ is the reduction in area at the cone due to the fiber formation, where $\Delta h(E)$ is the radius of the plate which is effectively approximated for the lost surface, as a function of the electric field, $E$. The second term $2 \pi \sigma h_{0}$ is the formation/nucleation energy of the fiber, where $\sigma$ is the line tension of the fiber. Note that $\Delta h(E)$ decreases with an 
a 1. Printing mixed solution

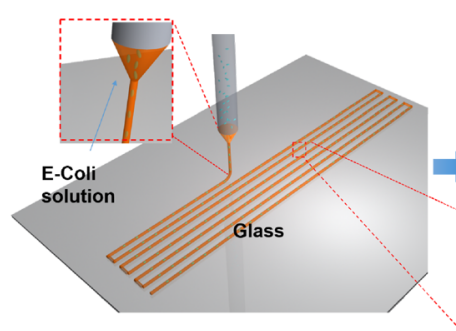

2. Covered by Agarose Gel

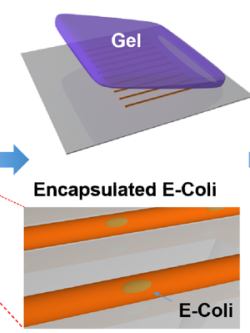

3. Dissolving carrier fibres

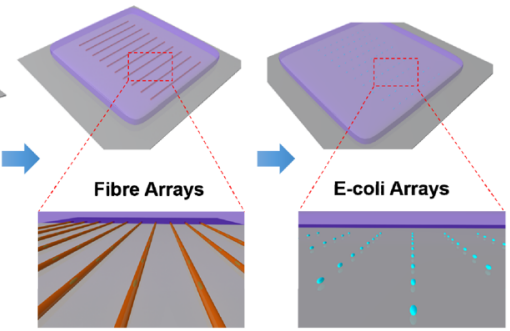

Bacteria culture

b
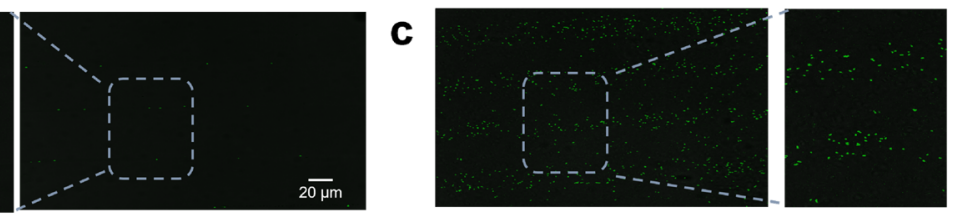

d
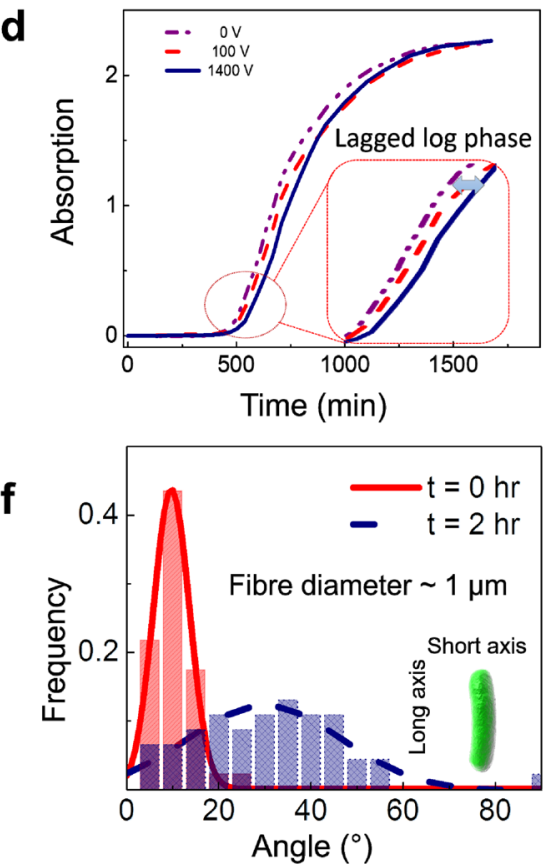

e

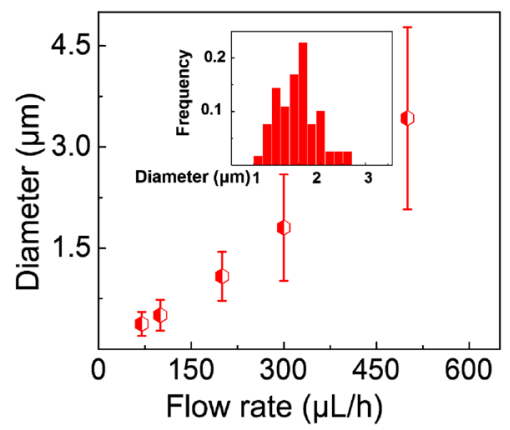

g

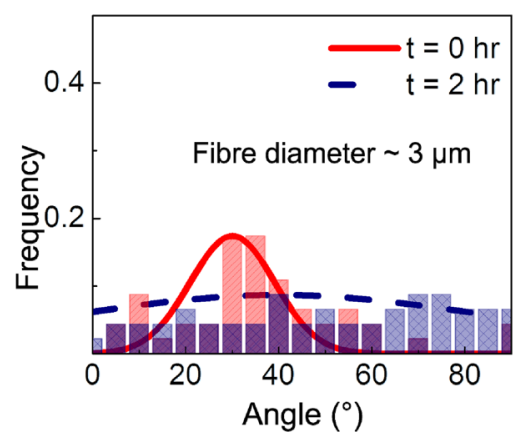

Figure 5. (a) Scheme showing the process of bacteria patterning using LEP. (b, c) Confocal images of E. coli arrays post deposition onto a glass coverslip. (d) Growth curves of the deposited bacteria and (e) line-width dependence on flow rate. Inset: histogram of the line width distribution at a flow rate of $300 \mu \mathrm{L} / \mathrm{h}$. (f) Bacteria orientation released from $1 \mu \mathrm{m}$ thick PEO fibers; straight line, at $t=0 \mathrm{~h}$; dashed line, after $2 \mathrm{~h}$ ' culture. (g) Bacteria released from $3 \mu \mathrm{m}$ thick PEO fibers. Particularly, the bacteria released from the $3 \mu \mathrm{m}$ fiber showed no peak in the angle distribution after 2 $\mathrm{h}$ of incubation, indicating the orientation is random.

increased electric field. By minimizing the total energy change of the system, $\Delta F$, with respect to the initial radius of the fiber, $h_{0}$, i.e., $\frac{\partial \Delta F}{\partial h_{0}}=0$, one can obtain, $h_{0} \propto \frac{\sigma}{\gamma}-\Delta h(E)$, showing that the initial fiber radius $h_{0}$ increases with the applied voltage due to the $-\Delta h(E)$ term. Meanwhile, we can also assume that the "poststretched" fiber radius follows the relationship of $h \propto h_{0} E^{-\alpha} \propto\left[\frac{\sigma}{\gamma}-\Delta h(E)\right] E^{-\alpha}$. Therefore, the dependence of fiber diameter on electric field depends on two competing factors $E^{-\alpha}$ versus $\frac{\sigma}{\gamma}-\Delta h(E)$. From our experimental results, it is shown that when the electric field is small, $E^{-\alpha}$ dominates how $h$ changes, actually decreases with the electric field; when the electric field is large, $\left[\frac{\sigma}{\gamma}-\Delta h(E)\right]$ dominates, and the fiber diameter $h$ increases with the electric field. Despite the voltage dependence, the histograms in Figure $4 a-c$ show that under a fixed operating condition, the fiber dimensions are relatively well controlled, i.e. for PS at 200-300 V, PVP at $120 \mathrm{~V}$ and $\mathrm{PEO}$ at $90 \mathrm{~V}$. It seems that the decrease of electrospinning voltage is at the cost of the enlarged variation of fiber width, thus further improvements are needed in order to enhance the uniformity of fiber dimensions in the low voltage electrowriting process. Using a gelatin solution, Figure 4e demonstrates that under the same voltage application, the average fiber diameter decreases when a smaller syringe tip is used. In order to give a comprehensive comparison, a brief summary on the experimental parameters and the as-spun fiber characteristics of selected current techniques for micronano scaled printing are shown Table S1. 
a

\section{Suspending fiber placements}

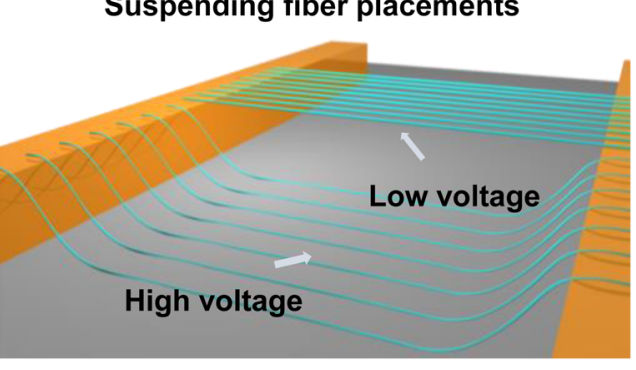

b
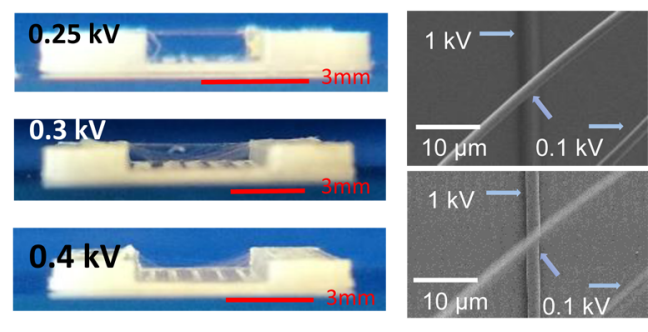
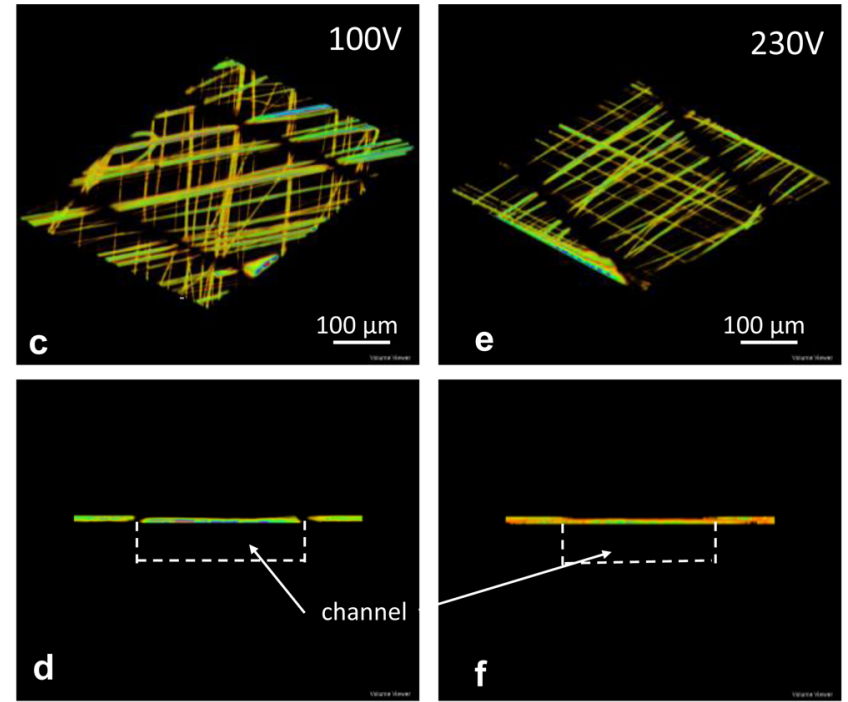

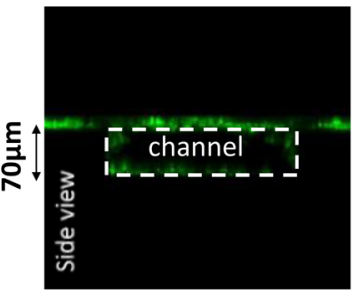

g

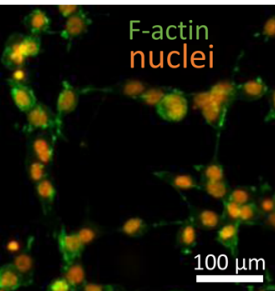

h

Low

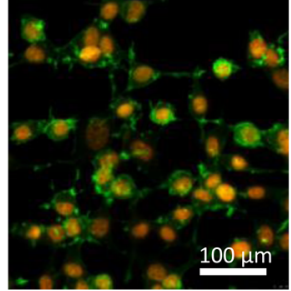

Fibre density

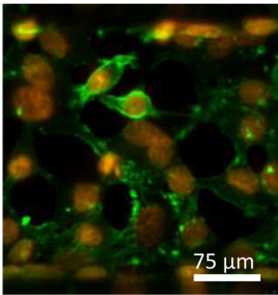

$75 \mu \mathrm{m}$

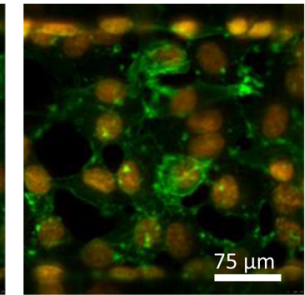

High

Figure 6. (a) Scheme of tuning fiber placement by adjusting the voltage application over a channel. (b) Optical images showing fibrils bridging experiments over a channel of $2 \mathrm{~mm}$ deep with a separation distance between 2.4 and $3.2 \mathrm{~mm}$. Fibers were fabricated using voltages of $0.25,0.3$, and $0.4 \mathrm{kV}$, respectively. As the voltage was increased, fibers were deposited onto the substrate underneath rather than being held in suspension. A transient region was also observed at a voltage of around $0.3 \mathrm{kV}$; on the right, SEM images show the PVP fibers were in suspension when fabricated using $0.1 \mathrm{kV}$, whereas laying on to the collector firmly when fabricated using $1 \mathrm{kV}$. (c, d) 3D reconstructed confocal images showing a layer of fibrous membrane network created using the LEP process at $100 \mathrm{~V}$. (e, f) 3D reconstructed confocal images showing a layer of fibrous membrane network created at $230 \mathrm{~V}$, with the membrane network more compact than the $100 \mathrm{~V}$ case. (g) Side view of the reconstructed confocal image of the cellcultured membrane within a microfluidic device. EA.hy926 cells were stained for cytoskeletons (F-actin, green) and nucleus (orange). (h) Top-view of the cell layer cultured on top of the suspending fibrous membrane over the microfluidic channel, showing that cellular morphology is modulated by the membrane fibril density.

Electrospinning Bacteria. The low-voltage (low electric field) operation provides a potential mild processing route for biological elements, e.g., living cells and proteins. One application is to directly pattern bacteria to study the influence of geometries on pathogenicity. Using far-field electrospinning, several groups have demonstrated the encapsulation of bacteria into nano- and microfibers; however, there are mixed reports on the bacteria cell viability. ${ }^{47-50}$ A further benefit of a lowered voltage operation is to widen the substrate choice for direct deposition (e.g., directly patterning on hydrogels or microfluidic devices). Thus, by providing a lower electric field intensity, the LEP technique brings unique advantages to meet the above two requirements. To demonstrate the feasibility for bacterial cell patterning, we encapsulated Escherichia coli (E. coli) into a carrier polymer which was deposited onto a glass substrate. Figure 5a shows the schematics of such a process. The E. coli suspension was first mixed with a carrier polymer (PEO-water, $10 \%$ in a culture media) to obtain adequate viscosity and conductivity for LEP. E. coli embedded PEO fibers with prescribed diameters were directly printed onto a glass coverslip. The sample was then covered with an agarose gel. After the carrier polymer was dissolved in the gel immediately, lines of E. coli arrays were remained for further cell culture.
Confocal images (Figure 5b, c) demonstrate the E. coli arrays patterned with the LEP technique. It was found that the number of $E$. coli multiplied significantly after $2 \mathrm{~h}$ incubation. One also observes that the bacteria were successfully configured into oriented arrays. Figure $5 \mathrm{~d}$ shows the growth curve of the deposited bacteria against a reference group where the bacteria were cultured in comparative conditions with the absence of an applied voltage, and a group of bacteria subjected to conventional high-voltage NFES processing. Each growth curve was fitted with a growth equation of $y=a /(1+$ $\left.\mathrm{e}^{-k\left(x-x_{\mathrm{c}}\right)}\right)$, where $x_{\mathrm{c}}$ indicates the time duration of the lag phase, and $k$ is the growth rate associated with the growth phase. These growth curves were employed to evaluate the effect of the LEP voltage application on the bacterial viability. The fitted parameters indicate that under the same experimental conditions, the onset of the lag-phase was delayed with increased voltage application, although the growth recovered after these initial periods. These experiments show that the use of low voltages may be important for certain applications to minimize the effects of voltages on the resulting bacteria behavior.

With a fixed applied voltage of $100 \mathrm{~V}$, tunable width of PEO fibers encapsulated with $E$. coli cells can be achieved by 
adjusting the solution flow rate during the LEP process (Figure 5e). For example, PEO fibers with $\sim 0.5 \mu \mathrm{m}$ width can be obtained by using a $100 \mu \mathrm{L} / \mathrm{h}$ flow rate; while a flow rate of 500 $\mu \mathrm{L} / \mathrm{h}$ can produce a fiber line width of $\sim 3.5 \mu \mathrm{m}$. It is found that the as-deposited fiber line width controls the subsequent bacterial orientation (described by the absolute value of the angle between the long axis of the E. coli cell and the printed line direction). With a $1 \mu \mathrm{m}$ thick PEO fiber, a narrow peak in the distribution shows that most of the orientation angle appears at $\sim 10^{\circ}$, which indicates that the bacteria embedded in the fibers were highly aligned (Figure 5f). This may be due to the narrow as-deposited fibers, which confined the bacterial cells (with a short body-axis of $\sim 0.5 \mu \mathrm{m}$ ) to their long bodyaxis. In contrast, when the fiber width increases to $\sim 3 \mu \mathrm{m}$, the bacterial orientation becomes more random (Figure $5 \mathrm{~g}$ ). Additionally, this thicker line provides more space to allow bacteria to stay close (e.g., two bacteria could be observed situated within a distance of $2 \mu \mathrm{m}$ from each other). It is to note that the orientation of $E$. coli cells also evolves with time. For example, for the sample of $1 \mu \mathrm{m}$ thick PEO fibers, at the moment the bacteria was printed, the orientation was mainly distributed between 0 and $20^{\circ}$. The peak of the distribution decreased and became more widespread after $2 \mathrm{~h}$ of incubation. For the sample of $3 \mu \mathrm{m}$ thick lines, the absence of the peak in the orientation distribution after $2 \mathrm{~h}$ of incubation indicates that the bacterial cells become randomly distributed.

Tuning Fiber Suspension, And Free-Standing Membranes. LEP also extends the application to construction of submicro- and microscale fibrous membrane architectures. In particular, this has applications for printing synthetic and biologically derived polymer scaffolds mimicking extracellular matrices (ECMs). Various 3D hybrid electrospun scaffold and direct-drawn free-standing fibrous net have been demonstrated. ${ }^{39,40,51-54}$ In this study, we show that by varying the subjected voltage magnitude, template-free and tunable suspension of individual fibers and membrane-net can be achieved using an aqueous polymer solution. First, we investigated the effect of voltage application on manipulating the suspending fibril structures. By electrospinning a 19 wt \% gelatin solution over PLA grids, Figure $6 \mathrm{a}$, b shows that when the applied voltage was tuned, one can adjust the electrospun fibers placement between in suspension and deposited at the base of the channel. A transient region is shown at around 300 $\mathrm{V}$ for a channel width of $3 \mathrm{~mm}$, at which fibers are partially suspended. SEM images illustrate that when electro-patterning was performed at a higher voltage (e.g., $1 \mathrm{kV}$ ), a fiber would firmly attach to the grounded substrate. When a lower voltage $(100 \mathrm{~V})$ was applied, the fibers can be "suspended" across over the gap as strands. We further explored the flexibility in controlling the fiber-substrate attachment by patterning fibril structures over PDMS (polydimethylsiloxane) with precasted channels. Figure $6 \mathrm{c}-\mathrm{f}$ shows reconstructed confocal images of fiber membranes fabricated with fluorescently labeled gelatin fibers. At $100 \mathrm{~V}$, a free-standing single membrane layer was formed, although the low voltage resulted in less attachment between membrane edges and the PDMS channel supporting sides. At $230 \mathrm{~V}$, thin and straight fibers were obtained. The voltage was sufficient for the membrane edges to attach firmly to the PDMS sides. We also observe a more compact membrane structure resulted from the $230 \mathrm{~V}$ fabrication compared to the $100 \mathrm{~V}$ case. Notably when a voltage is further increased to, for example, $500 \mathrm{~V}$ or greater, the fiber diameter was irregular with thick fibers forming over the PDMS channel.
The lack of controllability over fiber size makes it difficult to fabricate consistent samples.

Cell Morphology Study. Here, we use LEP to study the interaction between cells and fibrous structure mimicking an ECM network. Since LEP allows direct polymer-deposition on complex structure, we fabricated membrane-like fibrous networks on microfluidics made of PDMS, as shown in Figure 6g, h. The PDMS microfluidic device was casted with channels that was $120 \mu \mathrm{m}$ in width and $70 \mu \mathrm{m}$ in depth. To provide sufficient cell adhesion, the patterned PEO fibers were coated with collagen IV that is a native protein mostly found in the basement membrane. Human endothelial cell line EAhy926 was cultured on the fibrous membrane. By tuning the fiber density of the suspending network, one observes a gradual change in the cell morphology, as shown in Figure 6h. At low fiber density, where the pore-size of the fibrous network is larger than the size of a cell, cells are constrained, having their cellular processes elongating along the suspending fibers. There is little direct contact between cells. As the fiber density increases and the pore size is comparable to the cell size, cells can adhere to the rim of a "pore" in the fibrous network and fill the pores. In this case, there is increasing cell-cell contact through the fibers and cells spreading out more. As the fiber density further increases until the pore-size of fibrous network is about half the size of a cell, direct contact between cells increases significantly and there is an increased tendency in forming a confluent layer. It is important to note that, because the fibrous networks are freestanding instead of attaching to a substrate, the scaffold offers the freedom for potential cellular remodelling. It is known that cells can trans-migrate with pore sizes smaller than their nucleus (e.g., $7 \mu \mathrm{m}^{2}$, or $\sim 3 \mu \mathrm{m}$ diameter pore for cancer cells), ${ }^{55}$ thus we found cells were able to penetrate through the membrane pores in our systems and proliferated in the base of the channel. Overall, our experiment showed that LEP can be used as a one-step deposition procedure to fabricate micromter thick, suspending membrane decorating microfluidic devices. To this end, the LEP gives a potential route to the deposition of fibrous structures of a variety of materials for future organ-onchip applications.

\section{CONCLUSION}

In summary, we demonstrated a direct patterning method, LEP with a new, simple configuration that facilitates nano- and microscale fiber patterning with low voltages. It offers advantages over traditional electrospinning protocols in the range of usable materials and substrates, providing continuous deposition mitigating electric sparks. In order to guide the onset of fiber formation at voltages at around $100 \mathrm{~V}$, different types of initiators should be used depending on the polymer conductivity; to this end, the electrospinnable range of the polymeric solution concentrations is also widened. In the current work, we demonstrated fibril patterns of various polymers, directly deposited onto substrates such as hydrated agarose gel and PDMS with millimeter thicknesses. The wide range of voltage tuneability helps to build various suspending fibril structures. It also provides a platform to systematically study cellular behaviors under tailored physical environments. LEP shows potential in the field of fibril and membrane constructions, providing a flexible, adaptable route to printing living and viable bioelements, and electrically sensitive structures. 


\section{EXPERIMENTAL METHODS}

Setup. Our electrospinning setup consisted of an X-Y translational stage (PI micos, LMS- 60) to implement the patterning route and with a $\mathrm{Z}$ stage (Thorlabs, L490MZ/M) to tune the working distance accurately, a voltage source (built in house by Cambridge Electronic Department), a blunt metallic tip (BD Microlance) and a pump (World Precision Instruments, AL- 1000). The "initiators" could be silicon wafers (University Wafer) or glass slides with up to several millimeters thick.

Computation. The simulation of the electric field distribution when electrospin PEO solution was performed by a commercial finite element software package COMSOL. To simplify, it is assumed no charge dissipation for approximation. The relative permittivity of the PEO-water solution was assumed as 90 and the solution conductivity was set as $0.096 \mathrm{mS} / \mathrm{cm}$. The distance of the collector to the tip apex was fixed at $1 \mathrm{~mm}$ for the simulation, where the collector was chosen as $0.5 \mathrm{~mm}$ in thickness.

Sub-100 V Electrospinning. To demonstrate the generality of this technique, the solutions for electrospinning were prepared using various polymers and concentrations (in weight ratio). In detail, PEO $(\mathrm{Mw}=400 \mathrm{kDa}$, Sigma-Aldrich $)$ was prepared as 3, 4, 5, 6, 7, 8, 9, and $10 \%$ in water; PVP $(\mathrm{Mw}=600 \mathrm{kDa}$, Sigma-Aldrich $)$ was dissolved into ethanol to form $15,20,25$, and $30 \%$ solutions; PS $(\mathrm{Mw}=280 \mathrm{kDa}$, Sigma-Aldrich) was prepared as 20,25 , and $30 \%$ in DMF and gelatin (porcine skin Sigma-Aldrich) with 19 wt \% in an acetic acid and ethyl acetate combination were used as solutions for electrospinning. ${ }^{31}$ First, polymer solution was placed into a $1 \mathrm{~mL}$ plastic syringe (BD Plastipak) and a metallic tip (inner diameter between 0.2 to $0.4 \mathrm{~mm}$ ) was used to connect the voltage source. The substrate (either conducting or insolating materials) was placed under the tip location and two initiators were fixed on the substrate. During electrospinning, a flow rate of $200 \mu \mathrm{L}$ was fixed for all solutions to match a collector speed of $100 \mathrm{~mm} / \mathrm{s}$. Applied voltage ranged from 50 to $150 \mathrm{~V}$ for PEO-water solution, 50 to $200 \mathrm{~V}$ for PVP solution and 50 to $700 \mathrm{~V}$ for PS-DMF solution, and 100-150 V for gelatin solution. The working distance was set as $1 \mathrm{~mm}$, with an initiator height of $0.5 \mathrm{~mm}$ above the ground, for fabricating the fibers of which results are shown in Figure $4 a-c$, e. All the electrospinning experiments were conducted in room temperature and humidity conditions. The fabricated patterns were controlled by a LabVIEW program (National Instrument, USA) written in house.

Solution and Fiber Properties Measurement. All measurements were carried out at room temperature. Specifically, all the fiber diameters presented in this article were measured using both microscope and SEM, with a sample size more than 5 different points of single fiber, and 15 different fibers from the same experimental condition. Solution conductivity of the polymer solutions with different concentrations were measured using a conductivity tip (InLab 731 ISM, Mettler Toledo) and a rheometer (Physica MCR 501, Anton Paar) was used for viscosity measurement. The viscosity is calculated by extrapolating the viscosity curve to an angular frequency of 0 . Fiber diameter was measured from images taken by an optical microscope (ZEISS Axioplan) and a scanning electron microscopy (SEM, XL 30 sFEG, Philips and Zeiss EVO LS15); and analyzed by ImageJ.

Preparation of Bacterial Suspension. A loop of E. coli MRR stock was streaked onto a Luria broth (LB) agar plate and incubated at $37^{\circ} \mathrm{C}$ overnight. A single colony was then inoculated with $\mathrm{LB}$ culture media and grown overnight at $37^{\circ} \mathrm{C}$, with shaking at $200 \mathrm{rpm}$. This culture was transferred into fresh LB media (1:50) and incubated with shaking until stationary phase was reached $(12-14 \mathrm{~h})$ to obtain the microbial suspension, which contained $\sim 1 \times 10^{9}$ colony forming units per $\mathrm{mL}(\mathrm{CFU} / \mathrm{mL})$. The stationary phase culture was used to harvest bacteria by centrifugation at $1200 \mathrm{~g}$ for $5 \mathrm{~min}$, and then the bacterial pellet was resuspended in fresh $2 \times \mathrm{LB}$ media. Ampicillin was added into the LB agar plate and fresh liquid medium at $50 \mu \mathrm{g} / \mathrm{mL}$.

Suspending Fiber and Membrane Construction. The voltage applied ranges from 100 to $500 \mathrm{~V}$ to control the spatial position of the fibers. To fabricate suspending fiber configurations, we set the distance between the tip and collector at $3 \mathrm{~mm}$ and the thickness of initiator was $2.5 \mathrm{~mm}$. We note the effects of applied voltage on the fiber placements as shown in Figure 6b. Confocal (Leica) and SEM were used for characterizing the fiber and membrane morphologies. PVP ( $25 \%$ in ethanol), or gelatin (19 wt \% in an acetic acid and ethyl acetate combination) solutions were used to demonstrate the fibril patterning. It is noted that for suspending fibers and 3D construction, the fibers should be solidified rapidly to form solid struts. The freestanding strand was electrospun using various voltages shown in Figure 6. Microfluidics made from PDMS were used as the template for the fabrication of the membranes on the top of the channels.

EAhy926 Cell Culture. Human umbilical vein cell line (EAhy926) was cultured in Dulbecco's Modified Eagle Medium (DMEM) under $5 \% \mathrm{CO}_{2}$ at $37^{\circ} \mathrm{C}$. The cell medium DMEM was supplemented with $1 \%$ L-glutamine (Invitrogen), 10\% Fetal Bovine Serum (FBS, Sigma) and $1 \%$ Penicillin Streptomycin (Sigma). To achieve collagen IV coating, the microfluidic device was subjected to plasma treatment and collagen IV solution was added on the sample immediately afterward. The coated sample was incubated at $37{ }^{\circ} \mathrm{C}$ for $1 \mathrm{~h}$ followed by a thorough wash using DMEM to remove excess coating. The sample was immersed in DMEM overnight before cell seeding.

Cell Staining. EAhy 926 cells were fixed using $4 \%$ formaldehyde at $37{ }^{\circ} \mathrm{C}$ and the staining was performed under room temperature. The cells were permeabilized in $0.2 \%$ Triton $\mathrm{X}$ in PBS before being incubated with $4 \%$ bovine serum albumin (BSA) for $1 \mathrm{~h}$. Hoechst 33258 (Sigma) and Phalloidin 488 (Invitrogen) were used to stain the cell nuclei and cytoskeleton, respectively.

\section{ASSOCIATED CONTENT}

\section{Supporting Information}

The Supporting Information is available free of charge on the ACS Publications website at DOI: 10.1021/acsami.6b07797.

Table giving comprehensive comparison on the experimental parameters and the fiber characteristics resulting from selected current techniques for micro-nanoscaled fiber patterning (PDF)

\section{AUTHOR INFORMATION}

\section{Corresponding Author}

*E-mail: yysh2@eng.cam.ac.uk.

\section{Notes}

The authors declare no competing financial interest.

\section{ACKNOWLEDGMENTS}

This work has been supported by The Engineering and Physical Sciences Research Council (EPSRC) UK under the grant number EP/M018989/1, and a Royal Society Research Grant. We thank studentship and scholarship funding supports from the China Scholarship Council scholarship, the EPSRC doctoral training partnership, the Schlumberger Foundation, and the WD Armstrong Trust. We are also grateful for Dr Eileen Nugent's assistance in the bacteria cell culturing.

\section{REFERENCES}

(1) Liu, H.; Kameoka, J.; Czaplewski, D. A.; Craighead, H. G. Polymeric Nanowire Chemical Sensor. Nano Lett. 2004, 4, 671-675.

(2) Wang, X.; Kim, Y.-G.; Drew, C.; Ku, B.-C.; Kumar, J.; Samuelson, L. A. Electrostatic Assembly of Conjugated Polymer Thin Layers on Electrospun Nanofibrous Membranes for Biosensors. Nano Lett. 2004, 4, 331-334.

(3) Bowers, D. T.; Tanes, M. L.; Das, A.; Lin, Y.; Keane, N. A.; Neal, R. A.; Ogle, M. E.; Brayman, K. L.; Fraser, C. L.; Botchwey, E. A. Spatiotemporal Oxygen Sensing Using Dual Emissive Boron DyePolylactide Nanofibers. ACS Nano 2014, 8, 12080-12091. 
(4) Liu, C.; Hsu, P.-C.; Lee, H.-W.; Ye, M.; Zheng, G.; Liu, N.; Li, W.; Cui, Y. Transparent Air Filter for High-Efficiency PM2.5 capture. Nat. Commun. 2015, 6, 6205.

(5) Chattopadhyay, S.; Hatton, T. A.; Rutledge, G. C. Aerosol Filtration using Electrospun Cellulose Acetate Fibers. J. Mater. Sci. 2016, 51, 204-217.

(6) Guo, F.; Servi, A.; Liu, A.; Gleason, K. K.; Rutledge, G. C. Desalination by Membrane Distillation using Electrospun Polyamide Fiber Membranes with Surface Fluorination by Chemical Vapor Deposition. ACS Appl. Mater. Interfaces 2015, 7, 8225-8232.

(7) Pampal, E. S.; Stojanovska, E.; Simon, B.; Kilic, A. A review of Nanofibrous Structures in Lithium Ion Batteries J. J. Power Sources 2015, 300, 199-215.

(8) Chan, C. K.; Peng, H.; Liu, G.; Mcllwrath, K.; Zhang, X. F.; Huggins, R. A.; Cui, Y. High-performance Lithium Battery Anodes using Silicon Nanowires. Nat. Nanotechnol. 2008, 3, 31-35.

(9) Boas, M.; Gradys, A.; Vasilyev, G.; Burman, M.; Zussman, E. Electrospinning Polyelectrolyte Complexes. Soft Matter 2015, 11, 1739-1747.

(10) Grafahrend, D.; Heffels, K.-H.; Beer, M. V.; Gasteier, P.; Möller, M.; Boehm, G.; Dalton, P. D.; Groll, J. Degradable Polyester Scaffolds with Controlled Surface Chemistry Combining Minimal Protein Adsorption with Specific Bioactivation. Nat. Mater. 2011, 10, 67-73.

(11) Chow, L. W.; Armgarth, A.; St-Pierre, J.-P.; Bertazzo, S.; Gentilini, C.; Aurisicchio, C.; McCullen, S. D.; Steele, J. A. M.; Stevens, M. M. Peptide-Directed Spatial Organization of Biomolecules in Dynamic Gradient Scaffolds. Adv. Healthcare Mater. 2014, 3, 13811386.

(12) Hasan, A.; Memic, A.; Annabi, N.; Hossain, M.; Paul, A.; Dokmeci, M. R.; Dehghani, F.; Khademhosseini, A. Electrospun Scaffolds for Tissue Engineering of Vascular. Acta Biomater. 2014, 10, $11-25$.

(13) Kenawy, E.-R.; Bowlin, G. L.; Mansfield, K.; Layman, J.; Simpson, D. G.; Sanders, E. H.; Wnek, G. E. Release of Tetracycline Hydrochloride from Electrospun Poly(Ethylene-Co-Vinylacetate), Poly(Lactic Acid), and a Blend. J. Controlled Release 2002, 81, 57-64.

(14) Xue, J.; Niu, Y.; Gong, M.; Shi, R.; Chen, D.; Zhang, L.; Lvov, Y. Electrospun Microfiber Membranes Embedded with Drug-Loaded Clay Nanotubes for Sustained Antimicrobial. ACS Nano 2015, 9, $1600-1612$

(15) Lu, X.; Wang, C.; Wei, Y. One-Dimensional Composite Nanomaterials: Synthesis by Electrospinning and Their Applications. Small 2009, 5, 2349-2370.

(16) Gaharwar, A. K.; Mukundan, S.; Karaca, E.; Dolatshahi-Pirouz, A.; Patel, A.; Rangarajan, K.; Mihaila, S. M.; Iviglia, G.; Zhang, H.; Khademhosseini, A. Nanoclay-Enriched $\operatorname{Poly}(\varepsilon$-caprolactone) Electrospun Scaffolds for Osteogenic Differentiation of Human Mesenchymal Stem. Tissue Eng., Part A 2014, 20, 2088-2101.

(17) Luo, C. J.; Stoyanov, S. D.; Stride, E.; Pelan, E.; Edirisinghe, M. Electrospinning versus Fibre Production Methods: From Specifics to Technological Convergence. Chem. Soc. Rev. 2012, 41, 4708-4735.

(18) Reneker, D. H.; Yarin, A. L. Electrospinning Jets and Polymer. Polymer 2008, 49, 2387-2425.

(19) Reneker, D. H.; Yarin, A. L.; Fong, H.; Koombhongse, S. Bending Instability of Electrically Charged Liquid Jets of Polymer Solutions in Electrospinning. J. Appl. Phys. 2000, 87, 4531-4547.

(20) Li, D.; Wang, Y.; Xia, Y. Electrospinning of Polymeric and Ceramic Nanofibers as Uniaxially Aligned Arrays. Nano Lett. 2003, 3, $1167-1171$

(21) Xu, C. Y.; Inai, R.; Kotaki, M.; Ramakrishna, S. Aligned Biodegradable Nanofibrous Structure: A Potential Scaffold for Blood Vessel. Biomaterials 2004, 25, 877-886.

(22) Hochleitner, G.; Jungst, T.; Brown, T. B.; Hahn, K.; Moseke, C.; Jakob, F.; Dalto, P. D.; Grol, J. Additive Manufacturing of Scaffolds with Sub-Micron Filaments via Melt Electrospinning. Biofabrication 2015, 7, 035002.

(23) Visser, J.; Melchels, F. P. W.; Jeon, J. E.; van Bussel, E. M.; Kimpton, L. S.; Byrne, H. M.; Dhert, W. J. A.; Dalton, P. D.; Hutmacher, D. W.; Malda, J. Reinforcement of Hydrogels Using
Three-Dimensionally Printed Microfibres. Nat. Commun. 2015, 6, 6933.

(24) Huang, Y.; Bu, N.; Duan, Y.; Pan, Y.; Liu, H.; Yin, Z.; Xiong, Y. Electrohydrodynamic Direct-Writing. Nanoscale 2013, 5, 1200712017.

(25) Bu, N.; Huang, Y.; Wang, X.; Yin, Z. Continuously Tunable and Oriented Nanofiber Direct-Written by Mechano-Electrospinning. Mater. Manuf. Processes 2012, 27, 1318-1323.

(26) Huang, Y. A.; Duan, Y.; Ding, Y.; Bu, N.; Pan, Y.; Lu, N.; Yin, Z. Versatile, Kinetically Controlled, High Precision Electrohydrodynamic Writing of Micro/Nanofibers. Sci. Rep. 2014, 4, 5949-5949.

(27) Sun, D.; Chang, C.; Li, S.; Lin, L. Near-Field Electrospinning. Nano Lett. 2006, 6, 839-842.

(28) Huang, Y. Y. S.; Terentjev, E. M.; Oppenheim, T.; Lacour, S. P.; Welland, M. E. Fabrication and Electromechanical Characterization of Near-Field Electrospun Composite Fibers. Nanotechnology 2012, 23, 105305.

(29) Min, S.-Y.; Kim, T.-S.; Kim, B. J.; Cho, H.; Noh, Y.-Y.; Yang, H.; Cho, J. H.; Lee, T.-W. Large-Scale Organic Nanowire Lithography and Electronics. Nat. Commun. 2013, 4, 1773.

(30) Di Benedetto, F.; Camposeo, A.; Pagliara, S.; Mele, E.; Persano, L.; Stabile, R.; Cingolani, R.; Pisignano, D. Patterning of LightEmitting Conjugated Polymer Nanofibres. Nat. Nanotechnol. 2008, 3, 614-619.

(31) Xue, N.; Li, X.; Bertulli, C.; Li, Z.; Patharagulpong, A.; Sadok, A.; Huang, Y. Y. S. Rapid Patterning of 1-D Collagenous Topography as an ECM Protein Fibril Platform for Image. PLoS One 2014, 9, e93590.

(32) Zheng, G.; Li, W.; Wang, X.; Wu, D.; Sun, D.; Lin, L. Precision Deposition of a Nanofibre by Near-Field Electrospinning. J. Phys. D: Appl. Phys. 2010, 43, 415501.

(33) Huang, Z.-M.; Zhang, Y. Z.; Kotaki, M.; Ramakrishna, S. A Review on Polymer Nanofibers by Electrospinning and Their Applications in Nanocomposites. Compos. Sci. Technol. 2003, 63, $2223-2253$

(34) Zheng, Y.; Gong, R. H.; Zeng, Y. Multijet Motion and Deviation in Electrospinning. RSC Adv. 2015, 5, 48533-48540.

(35) Fuh, Y.-K.; Lu, H.-Y. Direct-Write, Microfiber-Based Template for Pattern Transfer of Aligned Metallic Microwires. J. Micro/ Nanolithogr., MEMS, MOEMS 2014, 13, 043014-043014.

(36) Taylor, G. Proc. R. Soc. London, Ser. A 1969, 313, 453-475.

(37) Chang, C.; Limkrailassiri, K.; Lin, L. Continuous Near-Field Electrospinning for Large Area Deposition of Orderly Nanofiber Patterns. Appl. Phys. Lett. 2008, 93, 123111.

(38) Bisht, G. S.; Canton, G.; Mirsepassi, A.; Kulinsky, L.; Oh, S.; Dunn-Rankin, D.; Madou, M. J. Controlled Continuous Patterning of Polymeric Nanofibers on Three-Dimensional Substrates Using LowVoltage Near-Field Electrospinning. Nano Lett. 2011, 11, 1831-1837.

(39) Nain, A. S.; Sitti, M.; Jacobson, A.; Kowalewski, T.; Amon, C. Dry Spinning Based Spinneret Based Tunable Engineered Parameters (STEP) Technique for Controlled and Aligned Deposition of Polymeric Nanofibers Macromol. Macromol. Rapid Commun. 2009, 30, 1406-1412.

(40) Nain, A. S.; Wong, J. C.; Amon, C.; Sitti, M. Drawing Suspended Polymer Micro-/Nanofibers using Glass Micropipettes. Appl. Phys. Lett. 2006, 89, 183105.

(41) Harfenist, S. A.; Cambron, S. D.; Nelson, E. W.; Berry, S. M.; Isham, A. W.; Crain, M. M.; Walsh, K. M.; Keynton, R. S.; Cohn, R. W. Direct Drawing of Suspended Filamentary Micro- and Nanostructures from Liquid Polymers. Nano Lett. 2004, 4, 1931-1937.

(42) Fong, H.; Chun, I.; Reneker, D. H. Beaded Nanofibers Formed During Electrospinning. Polymer 1999, 40, 4585-4592.

(43) Yu, J. H.; Fridrikh, S. V.; Rutledge, G. C. The Role of Elasticity in the Formation of Electrospun. Polymer 2006, 47, 4789-4797.

(44) Zander, N. Hierarchically Structured Electrospun Fibers. Polymers 2013, 5, 19.

(45) Huang, Y.-C.; Fan, P.-W.; Lee, C.-W.; Chu, C.-W.; Tsai, C.-C.; Chen, J.-T. Transformation of Polymer Nanofibers to Nanospheres 
Driven by the Rayleigh Instability. ACS Appl. Mater. Interfaces 2013, 5, 3134-3142.

(46) Cramariuc, B.; Cramariuc, R.; Scarlet, R.; Manea, L. R.; Lupu, I. G.; Cramariuc, O. Fiber Diameter in Electrospinning Process. J. Electrost. 2013, 71, 189-198.

(47) Connell, J. L.; Ritschdorff, E. T.; Whiteley, M.; Shear, J. B. 3D Printing of Microscopic Bacterial Communities. Proc. Natl. Acad. Sci. U. S. A. 2013, 110, 18380-18385.

(48) Salalha, W.; Kuhn, J.; Dror, Y.; Zussman, E. Encapsulation of Bacteria and Viruses in Electrospun. Nanotechnology 2006, 17, 4675.

(49) Nagy, Z. K.; Wagner, I.; Suhajda, A.; Tobak, T.; Harasztos, A. H.; Vigh, T.; Soti, P. L.; Pataki, H.; Molnar, K.; Marosi, G. Nanofibrous Solid Dosage Form of Living Bacteria Prepared by Electrospinning. eXPRESS Polym. Lett. 2014, 8, 352-361.

(50) Gensheimer, M.; Becker, M.; Brandis-Heep, A.; Wendorff, J. H.; Thauer, R. K.; Greiner, A. Novel Biohybrid Materials by Electrospinning: Nanofibers of Poly (ethylene oxide) and Living Bacteria. Adv. Mater. 2007, 19, 2480-2482.

(51) Zhang, D.; Chang, J. Electrospinning of Three-Dimensional Nanofibrous Tubes with Controllable Architectures. Nano Lett. 2008, $8,3283-3287$.

(52) Cai, S.; Xu, H.; Jiang, Q.; Yang, Y. Novel 3D Electrospun Scaffolds with Fibers Oriented Randomly and Evenly in Three Dimensions to Closely Mimic the Unique Architectures of Extracellular Matrices in Soft Tissues. Langmuir 2013, 29, 2311-2318.

(53) Park, S. M.; Kim, D. S. Electrolyte-Assisted Electrospinning for a Self-Assembled, Free-Standing Nanofiber Membrane on a Curved Surface. Adv. Mater. 2015, 27, 1682-1687.

(54) Kim, M. S.; Son, J.; Lee, H.; Hwang, H.; Choi, C. H.; Kim, G. Highly Porous 3D Nanofibrous Scaffolds Processed with an Electrospinning/Laser Process. Curr. Appl. Phys. 2014, 14, 1-7.

(55) Wolf, K.; te Lindert, M.; Krause, M.; Alexander, S.; te Riet, J.; Willis, A. L.; Hoffman, R. M.; Figdor, C. G.; Weiss, S. J.; Friedl, P. Physical Limits of Cell Migration: Control by ECM Space and Nuclear Deformation and Tuning by Proteolysis and Traction Force. J. Cell Biol. 2013, 201, 1069-1084. 